\title{
Quantum mechanical model of emotional robot behaviors
}

\author{
Martin Lukac \\ Portland State University \\ Portland, Oregon \\ lukacm@ece.pdx.edu
}

\author{
Marek Perkowski \\ Portland State University, \\ Portland, Oregon \\ mperkows@ee.pdx.edu
}

\begin{abstract}
In this paper the emotional model of the humanoid Cynthea (Cybernetic Networked Humanoid Emotional Agent) robot is presented. The robot is explained at two levels: the cognitive level is described by the CRL language, and the emotional level manipulates the language in a data independent way. An emotional mapping is introduced and is used to alter the language words using local energy-based rules. The robot is described as a set of functional agents, each acting on behalf of its own emotional function generating the whole robot emotional state. The cognitive level is applied to describe situations such as dialogues, interactions and behaviors specified in the CRL language. The emotional level is used as a mechanism to introduce behavioral changes in the robot's command execution. The interaction of these levels evolves in alterations to the desired behavior. To allow global effects on the language level with lower level behavioral variations we provide a model of robot-specific emotions based on quantum cellular automata.
\end{abstract}

\section{Introduction}

The emotional humanoid behavioral robotics is the area of research dealing with robots that are designed to communicate and interact with human users in a social environment. Various approaches have been explored such as biologically inspired models $[4,3,2,7]$ or more philosophically based such as $[8,5,9]$. Beside the technology-related problems such as the resource insufficiency (limiting the extent of the experimentation) and the very focused models (not allowing to perceive explored characteristics in known situations), the various emotional models are human based, and perhaps inadequate to be used on silicon devices. Moreover, in humanoid robots designed for human-robot social interaction, it is desired that the behavior is non repeating, surprising and appropriate. But the classical input-output driven schema does not allow more dynamical emotional expression patterns such as dialogue with appropriate be- havioral expressions. A dialogue is complicated not only because of the logical complexity but also because the robot must behaviorally stimulate the human into their dialogue. Thus it is not enough to attach emotional responses only to body states or dynamics but one must also provide a mapping that allows to use emotions on a higher, logical level.

The focus of this paper is on the emotional model of a single Cynthea robot that attempts to mimic emotions based on homeodynamic properties simulating a meta-level of the functional part of its own structure. In another words, the emotional model imitates the properties of biological functions and components. For example a human organ such as the stomach could be simulated on the functional level as a digestion system, or can be simulated as a multicellular system that when it is in its optimal state allows the best digestion. Thus, when such a simulated model is commanded to execute a given action, it will perform not only following the commands, but it will do this trying to create the most optimal behavioral expression of this command in its current state. The biological idea behind our model is to design devices that will use an internal state of robot's behavior that cannot be completely predicted but would still allows the robot's behavior to remain socially acceptable. The internal state of every functional agent is modeled as a State Machine and is extended to Quantum Finite Automata (QFA). This extension allows global quantum effects such as entanglement and superposition that are described and explored on the behavioral level. The CRL (Common Robot Language) language specifies the language (set of words) that the QFA accepts and is used as example in this paper. The hidden 'emotional component' is the set of all actions that the robot may use to express its emotional state by applying these 'emotional rules' as string rewriting to the cognitive-level words. We do not claim here to create a model explaining human emotions, our goal is only to design an interesting robot that mimics emotional behaviors. The Cynthea robot is represented using two embeded loops. First, an external loop represents the cognitive (control) level of the robot which describes the robot interacting with the environment using the CRL language. As the robot is designed for social 
interaction with humans, the first loop represents the robot perceiving environment through its sensors (including vision (face detection, recognition, tracking) and speech recognition), as well as processing the CRL scripts or commands and executing the given instructions (including speech generation). The second, internal loop represents the emotional level where the robot's components dynamically evolve, and interact using simulated quantum channels. Figure 1 represents the two loops. First, in the dashed frame presented is the emotional part that alters actions of the cognitive command from the bottom left of the figure. Second, the external loop is represented by the bottom of the figure.

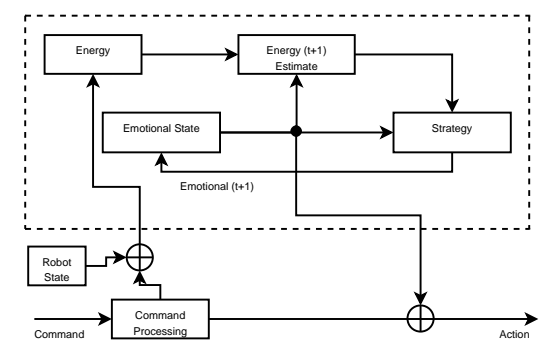

\section{Figure 1. Schematic diagram of one Emo- tional Agent in Cynthea controller.}

Using this model our research problem consist in finding how such emotional machines can be used to adapt social behaviors of talking head robots. This paper shows the details of the behavioral model of Cynthea in the following order. Section 2 introduces the theoretical basis of the emotional component. Section 3 describes an example of implementation. The Section 5 describes the quantum part of the machine. Section 6 explains how the emotions act on the robot at the global level. Finally conclusion discusses the model and the results obtained so far.

\section{Energy and Emotion}

The calculation of robot emotional expression (mood) from its emotional state is made using all of the emotional agents. A single emotional state can present a variety of behaviors; the mapping from emotion to behavior is very loose and allowing one-to-many relation between these two components; i.e. $R=\{X, Y, G\}$, where $\mathrm{X}$ and $\mathrm{Y}$ are the input and output set respectively and $G \subseteq X \times Y$ is the transition relation.

The Cynthea robot has six basic emotional variables: anger, happiness, melancholy, depression, fear and contentness. Anger, happiness, melancholy and depression are four basic states, while fear and contentness are dependent on the input and output state predictions. The mapping is executed in two steps. First the current emotional state is updated, then the expression of the state is selected (this implies the robot to change its current state). These two steps are referred to as energy and strategy. The four basic emotional states are mapped on the energy space, while the two input dependent states are mapped to the strategy space. This input dependent mapping is similar to the models used by $[3,9]$. From a more general point of view, the robot can be described as processing CRL scripts (cognitive level) and autonomously altering them (emotonal level) based on its current state. To represent the internal state of the robot, the energy (first step) allows to map any computational object or signals to a common energy space.

\subsection{Energy}

The energy function accepts the robot's state as its input and thus it does not depend directly on external robot inputs. This is because at a given time t, the state of the robot is represented by all functional elements of the robot (software, hardware and current commands). It uses the current energy state and the input (i.e. the functional input of the module - if any) to predict the next state of the energy after the execution of the given command. The energy function is defined upon the space $O^{d}$ as $E_{n}(p) \rightarrow 0,1 \quad \forall p \in R^{+}$, with p being any object having the $l^{2}-$ norm $\in R$.

When an object $\mathbf{p}$ (word in CRL) is processed, it is transformed into a set of energy coefficients that are then directly used in the computation of the next energy state. The predictions about the energy as well as the real agent's states are calculated using Gaussian distributions such as in equation 1. It allows for a given center $C$ (located in the middle of the emotional map), to calculate the direction to the desired optimum. Here, the center $\mathrm{C}$ plays a quantitative role: it determines the energy that the machine will try to match. Thus, it means that the energy will negatively reward any action that tends to move away from the center and will pay off for the opposite actions. The energy function is the prediction about the future evolution of the energy amount with respect to the current input, output and the state of the robot. Equation 1 shows the general shape of the energy distribution.

$$
e(o)=e^{e_{\text {diff }}}-\delta
$$

with $e_{\text {diff }}=\frac{-\left(c-x_{o}\right)^{2}}{2 r_{e}^{2}}$ and $\delta$ is a constant representing the continuous energy consumption. It is parameterized by several dynamical variables; $r_{e}$ - the radius and the center $c$. It is defined over a language $\lambda_{g} \subset C R L$ and a goal $\mathbf{X}$. The goal $\mathbf{X}$ is energy representation of the current state and $\Lambda$ is the subset of the CRL language. The goal is generally the task that the robot has to accomplish, for example any command given to the robot(agent) becomes its goal. The landscape represents the energy state evolution based on the language modification and goal representation. 


\subsection{Strategy}

The strategy function processes the value from the energy function domain and decides on the emotion expression. Strategy is defined as $S_{n}(p) R \rightarrow R$ and is unbounded on $\mathrm{R}$. The general form of the Strategy function is:

$$
s(o)=\frac{\sqrt{r_{s}^{2}+(e(o)-c)}}{r_{s}}
$$

Similarly to energy, the strategy process is defined over the language $\lambda_{e} \subset C R L$ and the strategy state $\mathbf{X}$. The strategy uses the energy output and the set of strategy parameters to generate a set of new emotional parameters that affect the command level. Similarly to the energy function, the strategy function is also parameterized by the radius $r_{s}$ and the center c. For each energy state, the strategy remaps the behavioral changes, according to either energy savings or strategy exploration.

The purpose of using an unbounded function is to allow a possibly 'infinite' decision space (eq. 2). Strategy is the function that the emotional processes use to modify both the emotional state and the functional state. Strategy affects the execution of the commands in the CRL language; i.e. the way various actions are executed by the robot.

\subsection{Robotic emotions}

To allow the manipulation of the basic emotional states the emotions are represented as vectors in the emotional space of limited dimension $O^{p}$. The energy function defined upon the space $O^{p}$ as $E_{n}(s) \rightarrow 0,1 \quad \forall s \in R^{+}$, with $s$ being the energy coefficient in the range of $\{0,1\}$. The energy coefficients represent the emotional state of the device. Operating on the state vector $\gamma$, the emotional state can be written in the form $E \times \gamma=\sum_{k=0}^{p-1} \beta e_{k} \times \gamma_{k}$, where $\beta$ is a constant. Energy function has a finite precision and thus is considered to be discrete and bounded on $R^{+}$in the range 0,1 .

The emotional state vector represents the degree of freedom that the emotional control has over the functionality of the device. This vector is represented as $\gamma_{i}=$ $\left\{\xi_{i}, s_{0}, \ldots, s_{n}\right\}$, where $\xi_{m}$ is the energy of the system and $s_{m}$ are the parameters of the device function ${ }^{1}$. The emotional machine represented by this vector, operates on its state variables using the state transition function $S$ (Strategy) that makes the state move towards the next state vector $v_{i}^{t+1}$. Because of the nature of hardware and software implementation, the transition function $\Theta$ is considered to be

\footnotetext{
${ }^{1}$ From the logic perspective, the cognitive level can be seen as the first order logic, while the emotional level represents higher order logic, allowing to manipulate, modify, reuse and transform the logic apparatus used on the cognitive level. For example for a servo, the task of the logic function is to create a physical change such as robot smiling. The task of the emotional level is to adjust this spatial change according to internal parameters.
}

time independent. The emotional machine can be characterized on the simplest level as the cognition-modification machine. The emotions in this machine modify cognition by rewriting words (including spoken English sentences) in the CRL language.

The equation 1 is mapped over two axis: $\frac{\partial E}{\partial x}$ and $\frac{\partial E}{\partial \lambda}$. Table 1 explains in more details the various combinations of the energy levels as they relate to goals and effects of language descriptions. As can be seen in Table 1, there are four cases given the slope of change of the partial derivatives of the Energy with respect to the Language and the Goal. This double dependence can be seen as a point $p(x, y)$ and a path from $p_{0}$ to $p(x, y)$. Because in the present situation the goal is fixed (it becomes fixed as soon as a given set of commands is processed) the emotion acts mainly over the language by rewriting words (character strings) in this language. In a similar way the strategy is mapped from the energy space to the decision making space (modifying the parameters of the system).

\section{Table 1. Goal and Language Effect}

\begin{tabular}{l|l|l|} 
& $\frac{\partial E}{\partial x}>0$ & $\frac{\partial E}{\partial x}<0$ \\
\hline$\frac{\partial E}{\partial \lambda}>0$ & Path Exploration & Path Exploration \\
& -- Goal Explo- & -- Goal Ex- \\
& ration 1) & ploitation 2) \\
\hline$\frac{\partial E}{\partial \lambda}<0$ & Path Exploitation & Path Exploita- \\
& -- Goal Explo- & tion -- Goal \\
& ration 2) & Exploitation 3) \\
\hline
\end{tabular}

In details these cases are:

1. $\frac{\partial E}{\partial \lambda}>0$ and $\frac{\partial E}{\partial x}>0$ - the case when both of the functions are increasing and the task representation is drifting toward the optimum (the state of the highest energy). This is the case of exploration of the description of path to the goal within the language $\lambda$ so as an exploration of the final condition; the goal.

2. $\frac{\partial E}{\partial \lambda} \frac{\partial E}{\partial x}<0$ - the case requires both components of different sign. This is the most common case, where both functions are of opposite sign and are generally related in a way such as: $\lim _{\lambda \rightarrow \infty} \frac{\partial E}{\partial \lambda}=-0$ and $\lim _{x \rightarrow \infty} \frac{\partial E}{\partial x}=0$ (and vice-versa).

3. $\frac{\partial E}{\partial \lambda}<0$ and $\frac{\partial E}{\partial x}<0$ - the case when both searches are only worsening the evaluation of the command.

\section{The emotional machine - an example}

To illustrate the concept of the emotional machine in more detail we define here a simple robot controller as a State Machine $G=\left\{Q_{g}, L_{g}, F_{g}\right\}$ that turns the eyelid controller left 
and right (we call it the $\mathrm{G}$ machine and it represents the Cognitive part of the robot). The set of states of this machine can be finite or infinite. The language $L_{g} \subset C R L$ operates on alphabet $\alpha_{g}$ that has $\mathrm{n}$ symbols, plus two symbols not in alphabet $L$ and $\diamond$. The language $L_{g}$ allows the maximum degree of freedom of the motion for a given servo as well as the commands to adjust the speed and the acceleration. Define Energy as Matrix $\Sigma$ of gradient values for every element in the state vector. For now, these are the ordinary matrix operators as will be seen later. The transition function is defined as $F \quad Q \times L \rightarrow Q$. The words of the language are CRL commands that can be interpreted by Cynthea software as robot's behaviors. Therefore all robot operations are described in this language. CRL was designed with only one level of recursion (one command can call any other command (including itself) only once) and thus can be used as the model of language that the emotional machine accepts. The diagram of the emotional State Machine is presented in Figure 2.

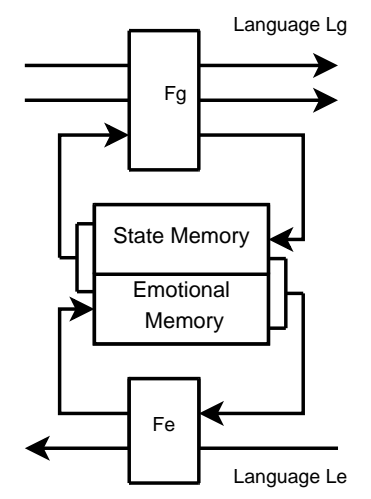

\section{Figure 2. The emotional State Machine.}

The hidden emotional component is similar to the above in the fact that it is also a FSM defined as $E=\left\{Q_{e}, L_{e}, F_{e}\right\}$ (the E machine). It operates on the set of the $Q_{e}$ states, in a way such that for an input word $\lambda$ and a state $\mathrm{q}$, the next state is given by $F_{e}(q, \lambda)$. Both machines $\mathrm{E}\left\{Q_{g}, L_{e}, F_{e}\right\}$ and machine $\mathrm{G}\left\{Q_{g}, L_{g}, F_{g}\right\}$ are parts of the same machine as can be seen on Figure 2.The top part (composed of the $F$ block - transition function - and part of the memory register called the state) represent the logic function used to interact with the environment and command the robot's autonomous behaviors. The bottom part including the block $F_{e}$ (emotion transition function and part of the register states) is the emotional part not connected to the environment directly. With $L_{g}, L_{e} \subset C R L$.

The whole machine can be defined as operating on the set of global internal states $Q=Q_{e} \times Q_{g}$. For the first submachine accepting $L_{g}$, the set of valid states is defined as $\left(\gamma, i_{a}, o_{a}\right)$, with $\gamma$ being the state transition function and $i_{a}$ and $o_{a}$ are set of valid inputs and outputs respectively.
This represents a one-to-one logic mapping space (the voice pitch set accepting $<$ pitch $>50</$ pitch $>$ command will always set the pitch to its median value). The second submachine accepting $L_{e}$ has a set of valid states defined by $\left(\gamma, i_{b}, o_{b}\right)$ but the input and output sequence language can be different. This can be written down as $L_{g} \bigcap L_{e} \neq\{\}$. The remaining sub-set $L_{g}^{-}$is a set of commands that the E machine will not be accepting. Also, as it can be expected that it holds that $\left(\gamma, i_{a}, o_{a}\right) \neq\left(\gamma, i_{b}, o_{b}\right)$ for $i_{a}, o_{a}, i_{b}, o_{b} \in L_{g}$. This implies, that for the robot executing a given command, the initial command is modified outside of the transition function $F_{e}$. The emotional submachine, despite the fact that it can only do what cognitive machine does, can however affect the machine $\mathrm{G}$ in some particular ways as implied from the definition of emotional functionality in section 2 . This particular influence of the emotional machine can be summarized in the following three cases:

a) emotional submachine can modify the time of execution such as: repeating the command, slowing or accelerating the command

b) emotional submachine can stop the command from being executed or can delete a command

c) emotional submachine can change the state of the machine $\mathrm{G}$ in order to produce a new command

Table 2 shows an example of an emotional machine that uses a two variable language to specify input and output and a single parameter emotional function. All possible sentences in the language are $\neg a \neg b, \neg a b, a \neg b$ and $a b$ and the emotional states are $c$ and $\neg c$. The rows represent the input specified by the energy encoding of the instruction and the columns represent the strategy alterations. This is one example how to translate the functions represented in equations 1 and 2. The minterms on variables $a, b$ in the second and third column of the Table 2 represent the gradient $\frac{\partial E}{\partial x}$ and thus they are favorable to the energy representation when increasing (the least favorable is 00 and the most favorable is 11). In the case of the Strategy component $\frac{\partial S}{\partial \Lambda}$, the optimal value is when the prediction about the actual output is equal to the actual command.

An example of emotional function is shown in Table 2. In this case, the emotional component is altered on all states but the $\{00, c\}$ state, where the robot does not change anything in any of the states $\{00,0\}$ and $\{00,1\}$. This could be compared to a state of easy fear, where the robot will be performing its task unless it is in its unfavorable energy state and also in its unfavorable strategy state. In another words, the predictions on the energy changes in the future after using this command are not favorable as well as the evaluated strategies for alteration of the language are unfavorable.

From the point of view of the language controlling the robot, the above functions must be translated to the robot 
Table 2. Emotional control easy fear and Language actions

\begin{tabular}{|c|c|c|c|c|}
\hline $\begin{array}{ll}\frac{\partial E}{\partial x} & \frac{\partial S}{\partial \Lambda} \\
\end{array}$ & c & $\neg c$ & c & $\neg c$ \\
\hline$\neg a \neg b$ & 00 & 00 & $\mathrm{r}, \mathrm{m}, \mathrm{i}$ & $\mathrm{r}, \mathrm{m}, \mathrm{i}$ \\
\hline$\neg a b$ & 01 & 11 & $\mathrm{~m}, \mathrm{~d}$ & $\mathrm{r}, \mathrm{m}, \mathrm{d}$ \\
\hline$a \neg b$ & 10 & 01 & $\mathrm{~m}, \mathrm{~d}$ & $\mathrm{~m}, \mathrm{~d}$ \\
\hline$\overline{a b}$ & 11 & 11 & $\mathrm{r}, \mathrm{m}, \mathrm{d}$ & $\mathrm{r}, \mathrm{m}, \mathrm{d}$ \\
\hline
\end{tabular}

instructions that control the robot behavior. From the behavioral level, using the function from Table 2 the robot will be able to stop executing any behavior because of its internal dissatisfaction. It is possible to create exhaustively all emotional functions of this type. However because the robot is created as a society of agents, mapping such a complex multilevel robot architecture might not make it possible to see the link between the low level emotions and the high level behaviors. However, looking closer at rules 1,2 and 3 , the output of the emotional function (Table 2) could be rewritten to one from Table 2 column 4 and 5. In the second and third column, the symbols are: $\mathrm{r}$ - repeat, $\mathrm{m}$ - modify, $\mathrm{i}$ - identity, $d$ - delete.

Thus the robot is expected to produce an unexpected result given a well defined sequence of commands. However, despite the fact of having a system that will achieve the given goal in its own optimal way, the emotional level must be limited with respect to the degree of freedom it has over the robot. For example the case $\bar{a} \bar{b}$ in Table 2 allows the robot for practically infinite command expansion. Also, because the application of the rewriting rules is recursive, the simple limitation of energy to properly describe emotions is not sufficient for the higher level behaviors. If the robot is in a happy mood and its strategy tells it to repeat, modify, identity then the result must be behaviorally different from the same type of strategy when the robot is in mood angry. But if the robot is happy it will never be in such a global state equivalent to for example angry because the composition of the mood is based on individual agent state.

\section{Emotional Control}

Given is $\Lambda$, a regular language with finite alphabet $\Gamma$. Beside the alphabet, two more characters $\angle$ and $\diamond$ are not in the alphabet, but are used to indicate the start and the end of the sequence of words. Let $\lambda_{m}$ be a valid word sequence in $\Lambda$, then emotional control is represented using the four basic string-operators (described above) and shown in Table 4:

To describe the emotional control, two main problems must be solved: the relation between the energy-strategy

\begin{tabular}{|c|c|c|} 
Operation & Strategy Space & Energy Space \\
repeat & $V_{r}\left(\lambda_{\mathbf{m}}\right) \rightarrow \lambda_{m} \lambda_{m}$ & $E-e_{v}$ \\
modify & $V_{r}\left(\lambda_{\mathbf{m}} \lambda_{\mathbf{m}}\right) \rightarrow \lambda_{l} \lambda_{m}$ & $E \pm e_{v}$ \\
identity & $V_{r}\left(\lambda_{\mathbf{m}}\right) \rightarrow \lambda_{m}$ & $E \pm e_{v}$ \\
delete & $V_{r}\left(\lambda_{\mathbf{n}} \lambda_{\mathbf{m}}\right) \rightarrow \lambda_{m}$ & $E+e_{v}$
\end{tabular} \mid

must be specified, the control of the changes by the strategy must be specified so that it could fit into the behavioral and social scheme. Thus, each time the energy is modified, the machine adjusts its activity using the above defined $V_{r}$ operators from Table $4^{2}$. And every time an input is processed, the machine readjusts itself by certain amount of change in the energy level.

\section{Quantum Emotions}

In this section, main properties of the quantum emotional model are explained and illustrated with examples simulated in software on a classical computer.

Quantum Finite Automaton (QFA) has been extensively studied and described in works $[10,1,6]$. QFA was shown to be at least as powerful as its classical counterpart (FSM). A QFA is defined by the tuple $\Gamma=\left\{Q, \Upsilon, \delta, q_{0}, Q_{a} c, Q_{r} j\right\}$, where $\mathrm{Q}$ is a finite set of states, $\Upsilon$ is the input alphabet, $\delta$ is the transition function such that $Q \times \Upsilon \times Q \rightarrow C^{0,1}$. The states $q_{0} \in Q^{\prime}, Q_{a} c \in Q$ and $Q_{r} j \in Q$ are the initial state, the set of accepting states and the set of rejecting states, respectively.

The emotional State Machine from Figure 2 can be now implemented as a cellular automata (CA) that has two types of interactions between its computational cells (agents being quantum automata). Every agent in this CA is half real dimension and half imaginary dimension machine, so that complex number signals that are used to represent these dimensions do not mix in the behavior space. Rather, the agents are directly communicating by the real inputs and outputs and general transformations are performed on a global level using quantum circuits and algorithms. The goal is to demonstrate some mechanisms causing that the quantum cellular automata are superior to classical CA when used in emotional robotics.

Some of our motivations to use concepts of quantum computing for the development of emotional robotics are based on these facts:

1. Quantum mechanics and quantum computing act on the energy level of physical matter. Thus these fields are the closest to a physical realization of the emotional robot.

\footnotetext{
${ }^{2} V_{r}$ represents the emotional function with respect to the command string and where $r$ is the emotional state
} 
2. Particular quantum effects such as superposition and entanglement can be used to generate unpredictable behavior, and thus quantum computing is well suited to implement an emotional machine used in human social environment. Observe that probabilistic effects and constrained behavior effects can be well modeled by the quantum measurement and quantum entanglement.

3. Because the emotional state is beside consciousness one of the most subjective and intimate matter to humans, it cannot be directly formalized or completely described. And as quantum computing based systems share similar principles about observability as this emotional definition, its use is directly deductible. However, the reader has to keep in mind that we do not claim that our model explains human emotions; our ambition is only to model it in a believable way.

From the practical standpoint, the language that the robot uses (CRL), has a real representation (words of CRL cannot be in superposition). In Cynthea, the application of quantum finite state automaton (QFA) corresponds to modifying the energy-strategy function using either the 1-way QFA or the 2-way QFA models[10,6] in the following way. In the 1-way QFA the machine reads the real input, then a unitary transform $\mathrm{V}$ is applied to the current state followed by measurement that affects the output. In the 2-way QFA, unlike in the classical QFA where the automaton is in contact directly with the input, the input and outputs are the states from the energy function that are never visible or observable. These states can be in superposition or they can be entangled while the data inputs to the machine remain always real.

To illustrate properties of quantum behaviors, assume a Hadamard Gate $\frac{1}{\sqrt{2}}\left(\begin{array}{cc}1 & 1 \\ 1 & -1\end{array}\right)$ and CNOT Gate $\left(\begin{array}{llll}1 & 0 & 0 & 0 \\ 0 & 1 & 0 & 0 \\ 0 & 0 & 0 & 1 \\ 0 & 0 & 1 & 0\end{array}\right)$. When multiplied as CNOT $* H \otimes W$ the resulting system is represented by the following matrix: $\frac{1}{\sqrt{2}}\left(\begin{array}{cccc}1 & 0 & 1 & 0 \\ 0 & 1 & 0 & 1 \\ 0 & 1 & 0 & -1 \\ 1 & 0 & -1 & 0\end{array}\right)$. The particularity of this system is the fact that as long as the system is not measured it is undetermined, however as soon as one of the bits is measured it sets the second one as well. Thus if two automata are in an entangled state and their outputs are measured, the results should coincide according to the entangled process and non local behavioral patterns will occur. Moreover, the emotional representation in the Cynthea model uses the complex phase [6] as representation. Using controlled gates such as CNOT, migrates the phase from the controlled bit onto the control bit [6], and thus shares the emotional state.

\section{Multi-Agent Emotional System}

To explain how the group behavior of these functional agents generates the emotion and moderates the behavior of the robot let us look at the robot structure. For this, assume a robot that has four CRL levels: the robot itself, the software part called eyes and mouth, the level of commands to this part and the actuator (a servo motor). As an example, the robot can be described with the following CRL command: < robot $><$ eyes $\mid$ mouth $><$ command $>$ parameters $<$ /command $></$ eyes $\mid$ mouth $></$ robot $>$. On the level of CRL rewriting (as executed by every level/module), the influence of the emotion goes from the most global level (the robot) to the most local one (a servo). Moreover, as explained in previous sections, the chances for rewriting on these levels depend on the displacements of their emotional states with respect to the optimal state. Thus in order for the command to be rewritten on certain level of the robot such as using the rule

\begin{tabular}{|c|c|}
\hline$<$ robot $>$ & $\langle$ robot $\rangle\langle$ robot $\rangle$ \\
\hline <eyes> & $<$ mouth $><$ eyes $>$ \\
\hline $\begin{array}{l}<\text { command }> \\
\text { parameters } \rightarrow \\
</ \text { command }>\end{array}$ & $\begin{array}{l}<\text { command }><\text { command }> \\
\text { parameters parameters } \\
</ \text { command }></ \text { command }>\end{array}$ \\
\hline </eyes> & $</$ mouth $><$ /eyes $>$ \\
\hline$/$ robot $>$ & $</$ robot $\rangle\langle/$ robot $\rangle$ \\
\hline
\end{tabular}

the energy requirement is much higher than when the rewriting is executed on the level of $<$ eyes $>$ such as using the rule.

\begin{tabular}{|c|c|}
\hline <eyes> & $<$ mouth $>$ \\
\hline$<\operatorname{command}_{0}>$ & $<\operatorname{command}_{1}>$ \\
\hline parameters $\rightarrow$ & parameters \\
\hline$<\operatorname{command}_{0}>$ & $\left\langle\right.$ command $_{1}>$ \\
\hline$<$ eyes> & $<$ mouth $>$ \\
\hline
\end{tabular}

From the behavioral point of view, the presented mechanism corresponds to various degrees of emotional comfort or discomfort and is explained as: in order for the robot to change the command from the highest levels of reasoning (global levels) the discomfort must be much higher than if the rewriting would occur on the command level (as in the example above). The generalized rewriting rules shown above are very general when looking at the detail level of each command. This is however not a parameter being studied here. Instead, such non specific operations will force the system to react to an internal change. 


\section{Conclusion}

The paper presented the emotional model developed for multi-body humanoid robot Cynthea. A scalable-languagearchitecture was introduced that describes how to design robots that interact with humans, based on the complexity dependent functional emotional model. An architecture of emotional machine has been proposed allowing to formulate precise framework for robotic behavior definition in order to express emotional behaviors that are generated on the lowest (body) level of the robot. The entire environment of the CRL-based model robot allows to precisely define behavior in robot-robot or human-robot interaction and thus is a tool for not only testing new robotic models and platforms but also theories of emotion-driven behaviors. The Cynthea framework provides yet only partial testing of our model. The robot has a software quantum simulator on the robotic level, and thus to run larger sets of robots more hardware must be assembled. Also the problem in obtaining a socially interactive robot is to provide a high quality speech recognition. Unlike the vision, the speech recognition should be as fluid as possible to ease the interaction. If the robot is slow to react the exhibit visitor will get bored and will leave. The problem of speech synthesis was not discussed here, and the reason is that currently the robot's speech engine is the rulebased ALICE system (itself based on the original ELIZA dialogue generator [11]). ALICE allows to express various speech alterations on the emotional levels, by describing various emotional states through the use of different AIML files. The ALICE system is convenient for simple dialogues, however for the completeness of the framework, it should be equipped with custom dialogue synthesizer that will allow to alter ALICE output text for local emotion related modifications.

\section{References}

[1] E. Bernstein and U. Vazirani. Quantum complexity theory. SIAM Journal of computing, pages 1411-1473, 1997.

[2] C. Breazeal. Designing Sociable Robots, . MIT Press, 2002.

[3] C. Breazeal and B. Scassellati. How to build robots that make friends and influence people. In Proceedings of IROS, pages 858-863, 1999.

[4] R. A. Brooks. Intelligence without reason. In Proceedings of the IJCAI, pages 569-595, 1991.

[5] D. Cañamero. A hormonal model of emotions for behavior control. Technical Report 97-06, Vrije Universiteit Brussel, 1997.

[6] J. Gruska. Quantum computing. Osborne/McGrawHill,U.S., 1999.

[7] T. Ogata and S. Sugano. Emotional communication between humans and the autonomous robot which has the emotion model. 1999.

[8] P. Serchuk, E. Sharlin, M. Lukac, and M. Perkowski. The vagueness of robot emotions. In Proceedings of the 36th International Symposium on Multiple-Valued Logic workshop on Post-Binary ULSI Systems, 2006.

[9] J. D. Velsquez. A computational framework for a emotionbased control. In Workshop on Grounding Emotions in Adaptive Systems, 1998.

[10] J. Watrous. On one-dimensional quantum cellular automata. pages 528-537, 1995.

[11] J. Weizenbaum. Eliza - a computer program for the study of natural language communication between man and machine. Communications of the Association for Computing Machinery, (9):36-45, 1966.

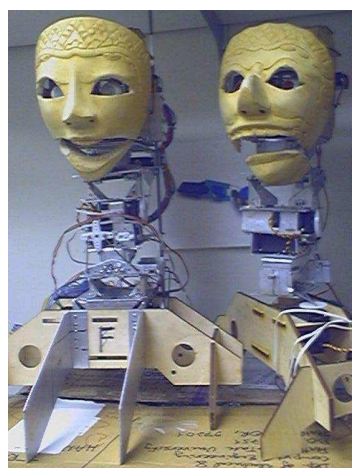

Figure 3. 